\title{
A PROTON-CYCLOTRON WAVE STORM GENERATED BY UNSTABLE PROTON DISTRIBUTION FUNCTIONS IN THE SOLAR WIND
}

\author{
R. T. Wicks ${ }^{1}$, R. L. Alexander ${ }^{2}$, M. Stevens ${ }^{3}$, L. B. Wilson III $^{4}$, P. S. Moya ${ }^{5,6}$, A. Viñas ${ }^{5}$, L. K. Jian ${ }^{4,7}$, D. A. Roberts ${ }^{4}$, \\ S. O'Modhrain ${ }^{2}$, J. A. Gilbert ${ }^{2}$, and T. H. Zurbuchen ${ }^{2}$ \\ ${ }^{1}$ Mullard Space Science Laboratory, University College London, Gower Street, London WC1E 6BT, UK; r.wicks@ucl.ac.uk \\ ${ }^{2}$ University of Michigan, Ann Arbor, MI 48109, USA \\ ${ }^{3}$ Harvard-Smithsonian Center for Astrophysics, Cambridge, MA 02138, USA \\ ${ }_{5}^{4}$ Code 672, NASA Goddard Space Flight Center, Greenbelt, MD 20771, USA \\ ${ }^{5}$ Code 673, NASA Goddard Space Flight Center, Greenbelt, MD 20771, USA \\ ${ }^{6}$ Department of Physics, Catholic University of America, Washington, DC 20064, USA \\ ${ }^{7}$ GPHI, Astronomy Department, University of Maryland, College Park, MD 20742, USA \\ Received 2015 February 10; accepted 2015 December 18; published 2016 February 23
}

\begin{abstract}
We use audification of $0.092 \mathrm{~s}$ cadence magnetometer data from the Wind spacecraft to identify waves with amplitudes $>0.1 \mathrm{nT}$ near the ion gyrofrequency $(\sim 0.1 \mathrm{~Hz})$ with duration longer than $1 \mathrm{hr}$ during 2008 . We present one of the most common types of event for a case study and find it to be a proton-cyclotron wave storm, coinciding with highly radial magnetic field and a suprathermal proton beam close in density to the core distribution itself. Using linear Vlasov analysis, we conclude that the long-duration, large-amplitude waves are generated by the instability of the proton distribution function. The origin of the beam is unknown, but the radial field period is found in the trailing edge of a fast solar wind stream and resembles other events thought to be caused by magnetic field footpoint motion or interchange reconnection between coronal holes and closed field lines in the corona.
\end{abstract}

Key words: instabilities - plasmas - solar wind - waves

Supporting material: audio files

\section{INTRODUCTION}

The solar wind is the tenuous plasma filling the heliosphere; it is emitted by the Sun and flows radially outward at speeds between 200 and $1000 \mathrm{~km} \mathrm{~s}^{-1}$. The solar wind is only weakly collisional; therefore, wave-particle interactions are important in determining the evolution of the proton distribution function. Energy can transfer from fields to particles and vice versa. Field-to-particle transfer of energy is important in explaining the nonadiabatic heating of the solar wind (Richardson \& Smith 2003), the anisotropic shape of thermal particle distribution functions (Kasper et al. 2002; Isenberg 2012), and the dissipation of large-scale turbulence (Goldstein et al. 1995). Particle-to-field energy transfer may arise from unstable particle distribution functions (Gary et al. 1984; Gary 1993, p. 127; Kasper et al. 2002; Hellinger et al. 2006; Matteini et al. 2007; Bale et al. 2009), for example, as a product of large-scale turbulence (Servidio et al. 2014), shock acceleration, or reconnection, and this transfer generates kinetic plasma waves (Bale et al. 2009; Wicks et al. 2013). These fundamental processes are determined by small-scale interactions. Thus, we need to observe magnetic and electric fields at ion-kinetic scales and frequencies, such as the proton gyroradius $\rho_{p}$ and gyrofrequency $\Omega_{p}$, in conjunction with detailed observations of particle distributions.

There are numerous different types of plasma wave that may interact with the proton distribution to exchange energy. The most commonly observed coherent waves close to ion scales in the solar wind are ion-cyclotron waves, which can be found in individual wave packets lasting just a few minutes (Jian et al. 2009), or in "cyclotron wave storms" lasting many hours (Jian et al. 2014). Cyclotron waves seen in the solar wind frame are left-hand-polarized electromagnetic plasma waves with frequencies close to the proton gyrofrequency in the plasma frame and wavevectors often close to the local magnetic field direction (quasi-parallel). Surveys of STEREO and $M E S S E N G E R$ spacecraft data have been used to identify and study ion-cyclotron-wave storms (Jian et al. 2010, 2014), although a complete description of how and why such storms happen is currently lacking.

We present a study of kinetic plasma waves and particle distribution functions observed by the Wind spacecraft. The Magnetic Field Investigation (MFI) instrument on the Wind spacecraft provides observations of magnetic field measured at $0.092 \mathrm{~s}$ cadence (Lepping et al. 1995), and the Solar Wind Experiment (SWE) instrument (Ogilvie et al. 1995) measures thermal particle distributions every $92 \mathrm{~s}$. Both instruments have operated since 1994, generating a data set of nearly $20 \mathrm{yr}$ of observations in the solar wind. In order to identify regions that exhibit wave-particle interactions, we look for magnetic-field fluctuations that display properties similar to those expected for proton-kinetic plasma waves. The challenge is to do this in an efficient way since $0.092 \mathrm{~s}$ cadence observations provide 950,400 samples per day and nearly $10^{10}$ samples to date. The waves of interest typically have a period of a few seconds, and so scanning through the data visually with resolution to observe the waves would entail many person-months of effort for proper identification.

Instead of visual analysis, we use audification, the direct translation of time-series data into audio, and listen to the data. Audification is a subdiscipline of sonification, the more general field of interpreting data as sound (Kramer 1994; Kramer et al. 2010). Audification has been used in many different studies in space plasma physics, particularly in the study of ionospheric and magnetospheric processes where several different phenomena have names derived from their audio properties, such as "whistler waves" (Barkhausen 1919, 1930), "lion roars" (Smith et al. 1969), and "hiss." Audification was also used to identify 
ring particle impacts on the Voyager 2 spacecraft as it passed Saturn (Scarf et al. 1982), and features of solar wind time series that were overlooked in a visual analysis have been detected using audification (Alexander et al. 2011, 2014; Landi et al. 2012). Audification allows us to survey the entire magnetic field data set for the year 2008 in a relatively short time and qualitatively compare the duration, amplitude, and clarity of events found easily. Audio playback is typically performed at rate of 44,100 samples per second. At this rate, 66.8 minutes of Wind MFI magnetometer observations last just $1 \mathrm{~s}$ in audio playback. The typical human ear can perceive sound between frequencies of 20 and $20,000 \mathrm{~Hz}$, with maximum sensitivity between 2000 and $5000 \mathrm{~Hz}$. This means that when the Wind MFI time series is converted into audio, frequencies in the original data between 0.005 and $5.5 \mathrm{~Hz}$ are audible, with maximum sensitivity between 0.5 and $1.25 \mathrm{~Hz}$. Therefore, the range of spacecraft frequencies that become audible when played through a speaker typically includes the inertial range of turbulence, $\Omega_{p}$, and $\rho_{p}$. The region of maximum auditory sensitivity is close to the range of frequencies at which we expect the cyclotron waves to occur, making the method particularly useful for identifying protonkinetic waves. The sampling frequency of the audio can be changed easily, allowing auditory analysis of different observational frequencies if required; however, for Wind MFI the sampling rate of 44,100 per second is ideal for the analysis presented below.

In this paper we select a case study event from 2008 November 4 , chosen because it is the most clearly audible wave signature in that year. The event is identified from the time series and spectral properties of the magnetic fluctuations and found to be a proton-cyclotron wave storm. Simultaneous plasma observations show that there is a dense proton beam, of which the density, temperature anisotropy, and drift speed correlate with the presence of the waves. Linear stability analysis, using a numerical solution of the Vlasov equation, shows that the proton distribution is unstable to the generation of cyclotron waves, consistent with our interpretation that the proton distribution function provides the free energy for the cyclotron waves.

\section{DATA}

The Wind spacecraft magnetometer (MFI; Lepping et al. 1995) provides at least $11 \mathrm{~Hz}$ cadence vector magnetic field observations nearly continuously for the entire mission lifetime. We chose to study the year 2008 because it is during the recent solar minimum, which should limit the number of complex events occurring in the year. During this period, the Wind spacecraft was orbiting the L1 Sun-Earth Lagrangian point and was continuously in the solar wind plasma flow.

\subsection{Audification}

The audification process isomorphically maps successive data samples to successive audio samples, making the data available for acoustic playback (Alexander et al. 2011). Data gaps in the original data are linearly interpolated so that the cadence of the samples remains fixed; thus, the audio accurately represents the time variations of the magnetic field, and data gaps therefore appear as low-volume "spectral holes" in the audio. In order to preserve the natural variability of the data, we scale the data linearly to ensure that all samples lie within the range $-1<x(t)<1$ :

$$
x(t)=2 \frac{B(t)-\min B}{\max B-\min B}-1,
$$

where $B$ can be any component of the magnetic field measured by MFI; we chose $B_{z}$, in GSE coordinates, for our study for reasons detailed below. Daily data sets were acquired from the SPDF Coordinated Data Analysis Web archive and combined into 12 audio files spanning 1 month of data each. Thus, the maximum and minimum values of the $B_{z}$ magnetic field data are taken once per month during 2008. Each month is then audified separately; thus, the exact scaling of the magnetic field is different from month to month. However, this will only affect the maximum volume of each audio file, not the frequency or response of the audio to any magnetic field fluctuations.

We chose the $\hat{z}$ GSE component of the vector for auditory analysis as it had no spacecraft-spin-induced noise (the $\hat{x}$ and $\hat{y}$ components have a low-amplitude residual spin noise in the audio that is of a similar frequency to proton-cyclotronresonant waves). One year of data for this single vector component contains roughly 330 million data points, which is approximately $2 \mathrm{hr}$ in length when audified as described above.

The resulting audio files were then assessed both auditorily and visually in the iZotope $\mathrm{Rx}^{8}$ software platform. iZotope $\mathrm{RX}$ generates a time series and spectrogram representation of the data that is scrolled through as audio playback occurs. A regular short-time Fourier transform with a window size of 2048 samples is used in the creation of the spectrogram. The turbulent solar wind presents itself auditorily as broadband noise with modulations in amplitude occurring at both small and large timescales, sounding somewhat like a flag waving in a strong wind. An example of an audio file containing the audification of the $B_{z}$ GSE component of the Wind MFI data from a relatively unremarkable region of turbulence is available for listening as an enhanced material to this article.

Through auditory and visual analysis, time periods were identified as potentially containing features of interest. A subset of seven periods containing clear auditory signatures with duration more than $4 \mathrm{~s}$ in the audio file (or 270 minutes in spacecraft time) were selected for additional analysis. An event on 2008 November 4 (DOY 309) was selected as the best candidate for this case study owing to activity that manifested as a long-duration, intense "whooshing" noise in the audified data. An audio file is available for listening as an enhanced material to this article.

\subsection{Case Study}

Time-series data from MFI over DOY 309 are shown in Figure 1. The top panel plots the time series of the three GSE components of $\boldsymbol{B}$ ( $x$ is blue, $y$ green, and $z$ red) and the magnitude of the vector (magenta). The second panel shows the magnetic field vector longitude (red) and latitude (blue) in GSE coordinates, with both being almost zero (black) over the majority of the day, indicating a radial field with the magnetic field vector pointing almost directly radially toward the Sun (where $B_{x} \sim|B|$ ). The third and fourth panels show the

\footnotetext{
8 iZotope $\mathrm{Rx}$ is software for audio editing and repair produced by iZotope, Inc.; the most recent version of the software is available at https://www. izotope.com/en/products/audio-repair/rx/.
} 


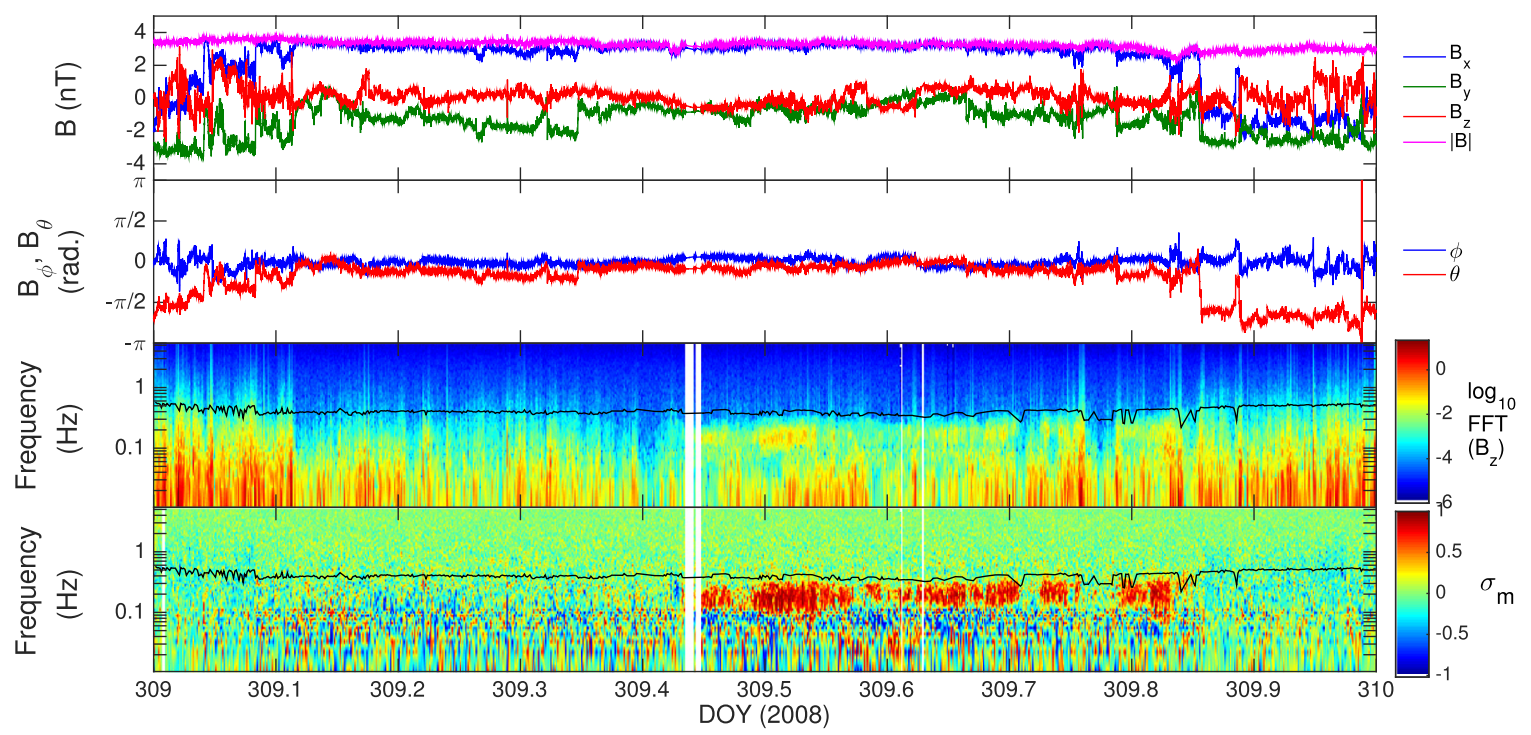

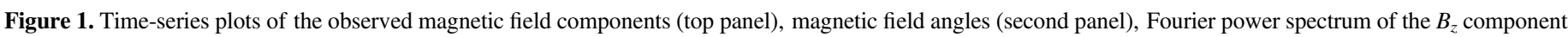

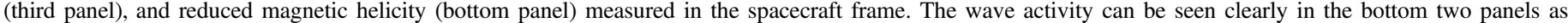

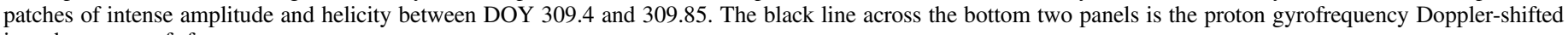
into the spacecraft frame.

windowed Fourier spectra of the $B_{z}$ GSE time series $\left(B_{z}\right.$ is the component used in the audio) and the reduced magnetic helicity of the magnetic field vector $\sigma_{m}$ :

$$
\begin{gathered}
\hat{B}_{i}=\frac{1}{\sqrt{T}} \int_{t-T / 2}^{t+T / 2} B_{i}(t) e^{-2 \pi i \omega t} d t, \\
P_{i j}=\hat{B}_{i} \hat{B}_{j}^{*}, \\
\sigma_{m}=\frac{2 \operatorname{Im}\left(P_{y z}\right)}{\sum_{i=j} P_{i j}},
\end{gathered}
$$

where $P_{i j}$ are the elements of the reduced power spectral tensor, $\operatorname{Im}()$ is the imaginary part of the tensor, and subscripts $i$ and $j$ define the components of the vector. We have used a window of $T=2048$ samples, equivalent to $184 \mathrm{~s}$, for the Fourier transform. We smooth the resulting power spectral tensor elements by taking the mean over a $10 \%$ fractional window about each frequency $f / \sqrt{1.1}<f<f \times \sqrt{1.1}$; this removes much of the high-frequency variability of the spectrum. For the purpose of plotting as time-frequency spectrograms, we then interpolate the smoothed spectra onto a logarithmically spaced frequency scale. Examples of the original and smoothed spectra can be seen in Figure 3, discussed below, and give an indication of the effect of the smoothing and interpolation process. We also note that, because the field in the interval containing the waves is radial, the magnetic helicity $\sigma_{m}$ contains the entire helicity of the magnetic field waves, with the other two components of the helicity being approximately zero.

The wave activity can be seen as a band of increased amplitude in the Fourier spectra and strong helicity signals in the frequency range $0.1<f<0.5 \mathrm{~Hz}$ in the spacecraft frame between DOY 309.45 and 309.85 in Figure 1. The black line in each of the bottom two panels of Figure 1 shows a rough estimate of the Doppler-shifted proton gyrofrequency $f_{\mathrm{sc}}^{-}$in the frame of the spacecraft:

$$
f_{\mathrm{sc}}^{ \pm}=\frac{\Omega_{p}+\boldsymbol{k} \cdot \boldsymbol{V}_{\mathrm{sw}}}{2 \pi} \approx \frac{\Omega_{p}}{2 \pi}\left|\left(1 \pm \frac{V_{\mathrm{sw}}}{V_{A}}\right)\right|,
$$

for a wave propagating parallel (+) or antiparallel (-) to the local mean magnetic field with phase speed equal to the Alfvén speed $V_{A}$ (Jian et al. 2009). We have purposefully not used a more precise dispersion relation to solve for $f_{\mathrm{sc}}^{ \pm}$because the precise direction and magnitude of $\boldsymbol{k}$ cannot be calculated with certainty directly from the data and the error in the velocity and changing magnetic field direction make the uncertainty in the result large. The resonant activity is at a slightly lower frequency than $f_{\mathrm{sc}}^{-}$, meaning that the wave either has a lower frequency, has a higher phase speed, or is propagating at a slightly oblique angle to the background magnetic field in the plasma frame. This is consistent with previous results from Jian et al. (2009, 2010, 2014).

The SWE reduced velocity distribution functions measured at the same time as the magnetic field are fitted to two biMaxwellian velocity distributions, one for the core of the proton distribution and one for a suprathermal proton beam component. This fit is used to calculate velocity, density, temperature, and temperature anisotropy relative to the local magnetic field direction for each population. An example of a field-parallel slice of a fitted distribution is shown in the left panel of Figure 2. The density, radial velocity, and temperature anisotropy of these two fits are plotted in the first, second, and third panels on the right side of Figure 2, respectively. The fourth panel shows the drift of the beam component away from the Sun relative to the core, along the magnetic field direction, normalized by the local Alfvén speed. This speed is used to calculate the kinetic energy density of the beam relative to the core, in Alfvén-normalized units in the fifth panel. The biMaxwellian fit of the SWE distribution functions shows that, at the same time as the MFI data show high-amplitude waves in the magnetic field, the density and kinetic energy of the beam increase. 

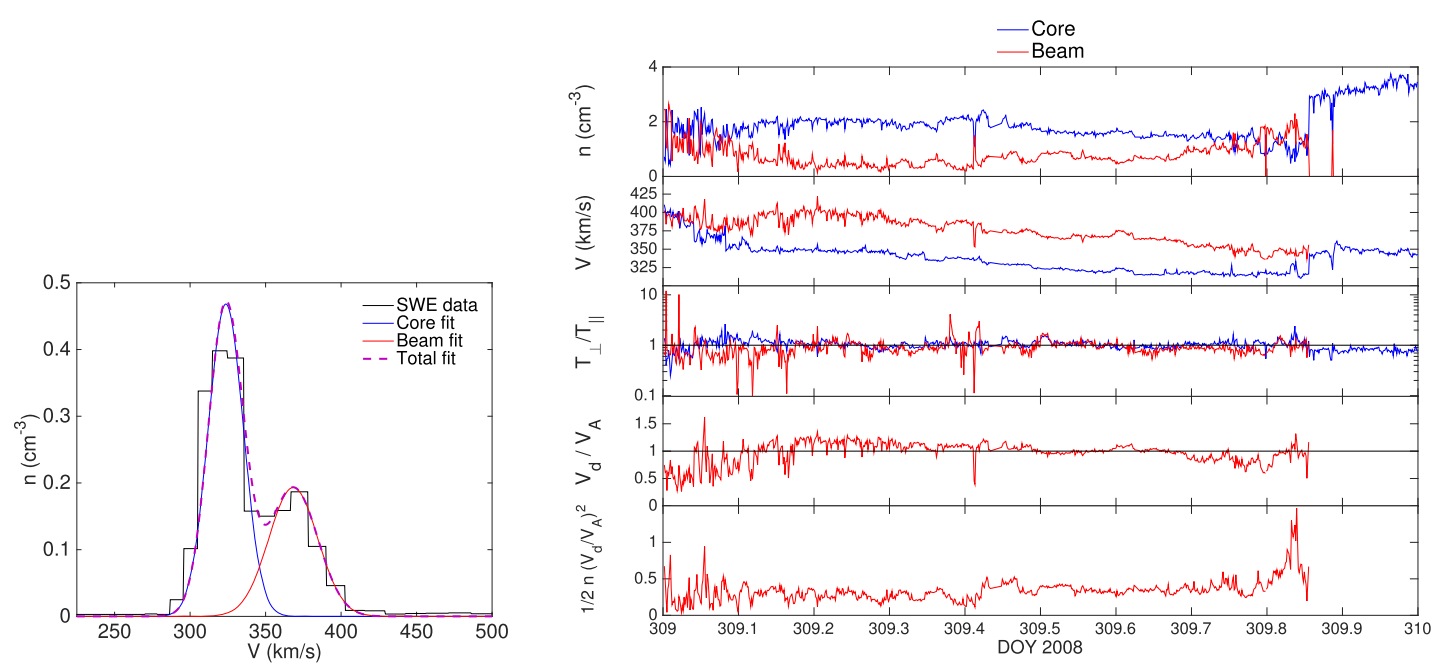

Figure 2. Left panel: example of the bi-Maxwellian fitting of the core and beam in the look direction parallel to the local magnetic field, taken from midday (DOY 309.5). The right panels show time-series plots of proton core and beam density (first panel), velocity (second panel), temperature anisotropy $\left(T_{\perp} / T_{\|}>1\right)$ (third panel), proton beam drift speed relative to the core normalized to the Alfvén speed and the related kinetic energy (fourth panel), and Fourier power spectrum of the $B_{z}$ component (fifth panel). The most intense wave activity seen in the bottom two panels of Figure 1 appears to be contemporaneous with increases in the density, temperature anisotropy, and kinetic energy in the beam distributions, particularly between times 309.45 and 309.52 .

\section{ANALYSIS}

Having identified a long-duration audio event as summarized in Figure 1 and identified that there are associated changes in the thermal proton distribution in Figure 2, we now perform a detailed analysis of the magnetic fluctuations composing the event and the associated thermal proton distributions to identify the wave modes and their likely cause. The reduced nature of observations made by single spacecraft (meaning that the plasma sampling is restricted to a one-dimensional time series from which spatial and temporal changes cannot be separated) rules out identifying the waves by comparing dispersion relations or wave propagation speeds in the plasma rest frame and makes the observation of an accurate wavevector $(\boldsymbol{k})$ impossible.

\subsection{Magnetic Field}

We begin by looking at the spectral and time-series characteristics of the fluctuations in Figures 3 and 4. The top panel of Figure 3 shows the trace power spectral density of the vector magnetic field observations from a period preceding the wave event (Background, 309.3484-309.3887 or 08:21:41 to 09:19:44, red) and a similar length of time during the most intense part of the wave event (Waves, 309.5-309.5403 or 12:00:00 to 12:58:02, blue). In each case the lighter-colored lines show the unsmoothed Fourier transform of the $1 \mathrm{hr}$ time series; the darker-colored lines show the smoothed and interpolated spectra resulting from our Fourier method. The background spectrum shows a steeper than $f^{-5 / 3}$ spectral scaling (black dot-dashed line) consistent with turbulent scaling in radial field intervals (Horbury et al. 2008; Podesta 2009; Wicks et al. 2010). The wave spectrum has a similar turbulent spectral scaling but also has a very prominent peak in the frequency range $0.06<f<0.3 \mathrm{~Hz}$ that rises to two orders of magnitude above the background spectrum. The Dopplershifted proton gyrofrequencies in the spacecraft frame $f_{\mathrm{sc}}$ are plotted as the green vertical dashed (wave propagation toward the Sun $\Omega_{p}^{I}$ ) and dot-dashed line (wave propagation away from the Sun $\Omega_{p}^{O}$ ). Frequencies higher than $f \sim 0.4 \mathrm{~Hz}$ are affected by instrument noise. To estimate the noise floor of the MFI, we have used the values quoted for the rms value of uncertainty in Lepping et al. (1995) $\left(12.1 \times 10^{-3} \mathrm{nT}\right)$ and applied a spectral scaling to lower frequencies of $f^{-1}$. The result is plotted as a black dashed line and is slightly higher than the observed spectra, which are also nearly identical to one another for $f>0.4 \mathrm{~Hz}$, strongly implying that noise is the source of the signal but that our estimation of the spectrum of the instrument noise floor is slightly pessimistic.

The second and third panels in Figure 3 show the reduced magnetic helicity $\sigma_{m}$ (Equation (4)) and magnetic variance anisotropy:

$$
\frac{\delta B_{\|}}{\delta B_{\perp}}=\frac{P_{z z}}{P_{x x}+P_{y y}},
$$

where the magnetic field has been rotated into a field-aligned coordinate system with the $\hat{z}$ direction along $\boldsymbol{B}_{0}$ and the $\hat{x}$ and $\hat{y}$ directions perpendicular (see, e.g., Wicks et al. 2012); the average background field over the hour-long period of each spectrum is used to define $\boldsymbol{B}_{0}$. The horizontal lines show $\sigma_{m}=0$ and $\delta B_{\mid} / \delta B_{\perp}=0.5$, which are the values expected for white noise in the components of $\boldsymbol{B}$. The background spectrum (red) shows no coherent reduced magnetic helicity signature (second panel) and is transverse (third panel) but decreasingly so with increasing frequency, typical for turbulent solar wind fluctuations (Podesta \& TenBarge 2012; Kiyani et al. 2013). The wave spectrum, however, shows high values of reduced magnetic helicity in the range $0.06<f<0.3 \mathrm{~Hz}$ and is more transverse over the same range compared to the background spectrum. Combining the results of Figure 3, we see that the trace, helicity, and variance anisotropy spectra show that there is a high-amplitude, helical, transverse signal in the magnetic field at frequencies $0.06<f<0.3 \mathrm{~Hz}$, just below the Dopplershifted proton-cyclotron frequency in the spacecraft frame during the wave interval, but not in the background interval.

Hodograms of the magnetic field in GSE coordinates and in the minimum variance coordinate system for a few wave 


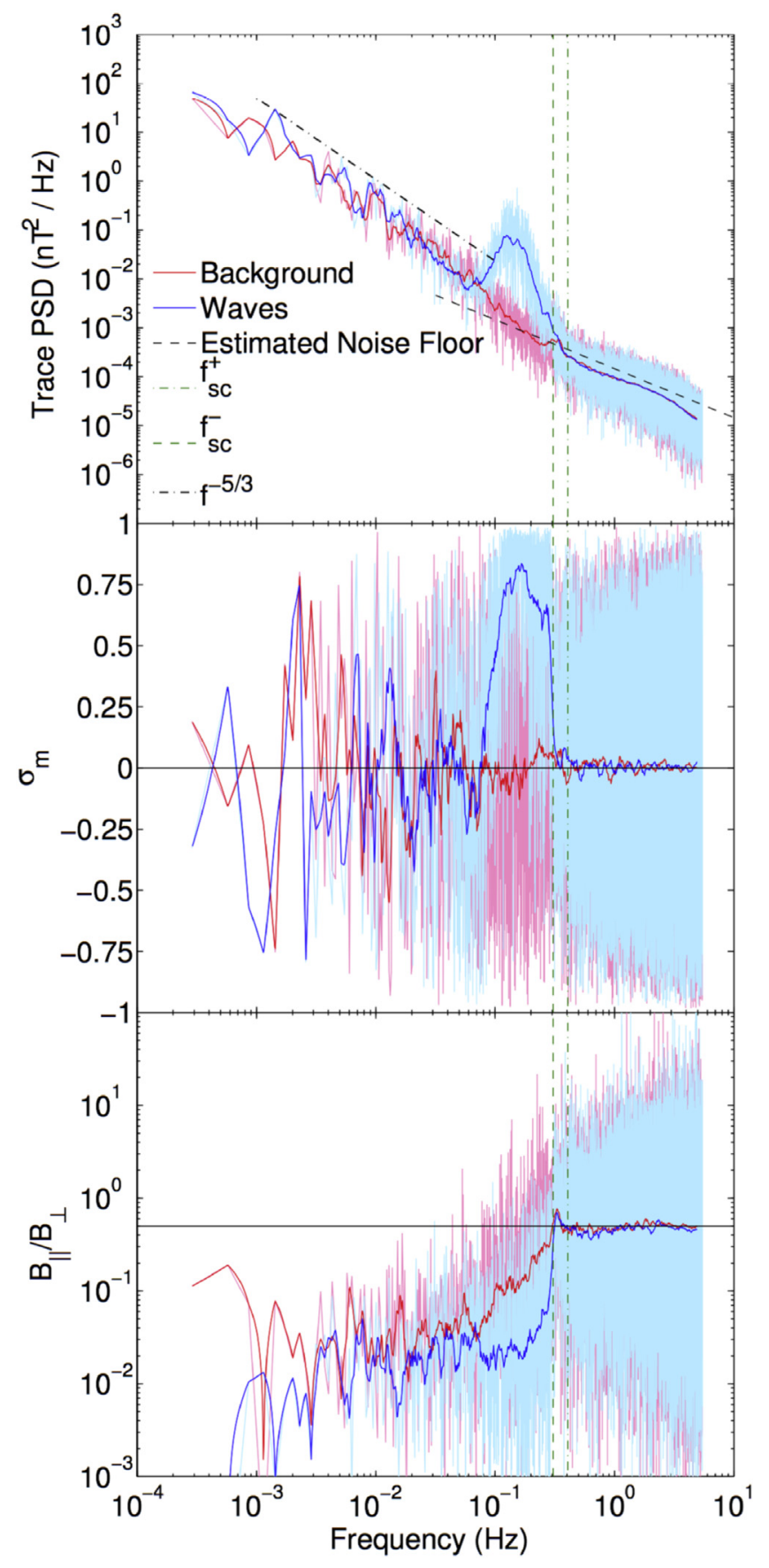

Figure 3. Spectral properties of the magnetic field during two different hourlong intervals of DOY 309. The first interval (unsmoothed raw Fourier transform in light red, final smoothed spectrum in red, $309.3484<t<309.3887$ ) is a quiet period of radial field before the wave event begins. The second interval is during an intense part of the wave event (unsmoothed raw Fourier transform in light blue, final smoothed spectrum in blue, $309.5<t<309.5403$ ). The top panel shows the trace magnetic field spectrum, the middle panel shows the magnetic helicity $\sigma_{m}$, and the bottom panel shows the magnetic variance anisotropy $\frac{\delta B_{\|}^{2}}{\delta B_{\perp}^{2}}$.

periods are shown in Figure 4. The analysis follows the procedure of Wilson et al. (2009) where the raw time-series data are bandpass filtered around the range of frequencies of the peak wave amplitude $0.06<f<0.3 \mathrm{~Hz}$. The resulting waveforms are shown in the left column of the left-hand set of six panels in Figure 4; a clear sinusoidal oscillation can be seen in all three components, with a maximum amplitude of $0.27 \mathrm{nT}$. Minimum variance analysis is performed on the filtered data, and one eigenvalue ( $\lambda_{3}$ in Figure 4$)$ is found to be much smaller than the other two, indicating a well-defined minimum variance direction. The time series of the three minimum variance coordinate system components of the filtered magnetic field are plotted in the second column of three panels and show clearly sinusoidal fluctuations in the intermediate and maximum variance directions, the eigenvalues of which are nearly equal, and no clear signal in the minimum variance direction. In the right-hand set of panels of Figure 4 we plot hodograms of the magnetic field in the three intersecting normal planes of each coordinate system. In the left column of panels the GSE coordinate representation shows a left-handed fluctuation in the spacecraft frame about the mean magnetic field direction, which is very close to the GSE $\hat{x}$ direction. The minimum variance analysis (right column of panels) shows a nearly circularly polarized wave. There is a sign ambiguity in the minimum variance direction, and so it is impossible to determine whether the wave is left- or righthanded in the plasma frame from the observations, which are in the spacecraft frame (Wilson et al. 2009, 2013).

Taking the results shown in Figures 1, 3, and 4 together, we have observed long-duration, transverse, coherent magnetic field fluctuations close to, but at a lower frequency than, the proton gyrofrequency Doppler-shifted into the spacecraft frame, which is left-handed in the spacecraft frame with a minimum variance direction at an angle of $13^{\circ}$ to the local magnetic field direction. These results indicate that it is likely that the waves are proton-cyclotron waves (Jian et al. 2009, 2010, 2014).

\subsection{Proton Distributions}

Given the presence of long-duration, high-amplitude, cyclotron waves, we investigate the proton distribution function next, to determine whether the wave activity is generated by the local thermal particle distribution and, if so, whether any unstable modes would generate cyclotron waves.

\subsubsection{SWE Observations}

Figure 2 indicates a close correlation between the beam kinetic energy and the presence of the waves. There are many ways that a non-Maxwellian proton distribution can generate waves. To identify which mechanism may be causing the waves, we first look at the magnetic-field-parallel cut of the reduced proton distribution observed by SWE to see whether there is a positive gradient between the core and beam distributions that could be a source of free energy for the beam instability. The top two panels in Figure 5 show the trace of the windowed Fourier transform and the magnetic helicity, showing the presence of the waves, identical to the bottom panels in Figure 1. The third panel shows the charge flux in amps on the sector of the SWE instrument detector most closely aligned with the local $B_{0}$ for each measurement. The vertical axis shows the speed of the incoming particles and the horizontal axis the time. The peak of the detected charge flux appears as a red line; a secondary peak can be seen as a fuzzy red area at speeds just less than $400 \mathrm{~km} \mathrm{~s}^{-1}$, particularly between times 309.45 and 309.55, where the magnetic field waves have the highest amplitudes. The bottom panel shows the gradient of the observed distribution functions. The peak of 
Date: $2008-11-04$

SCET : $12: 47: 51.0025-12: 48: 10.4145$

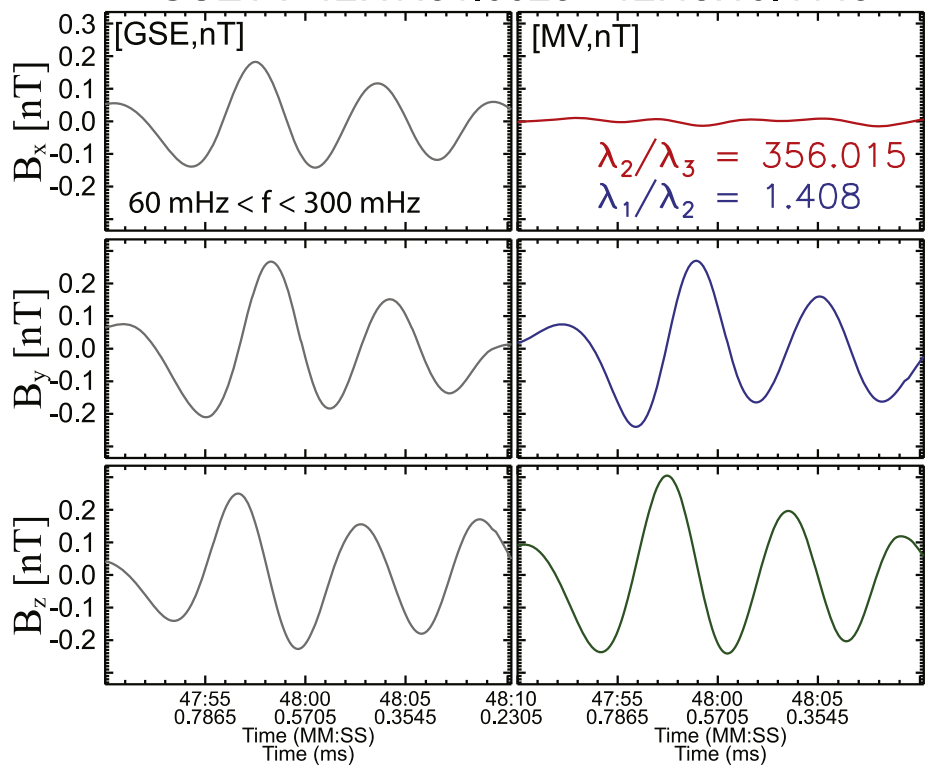

$k_{\mathrm{GSE}}=\langle+0.810,-0.452,-0.373\rangle$
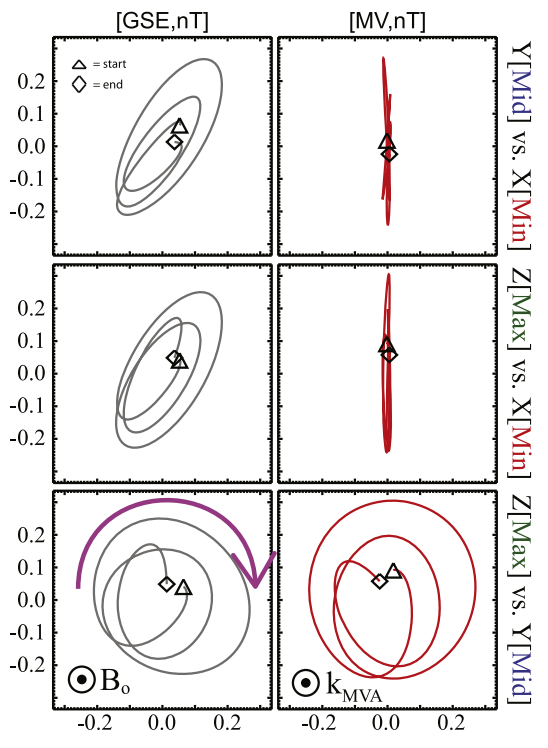

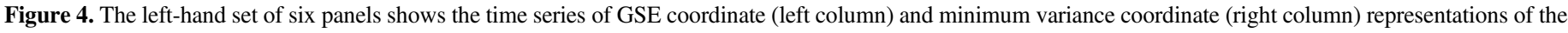

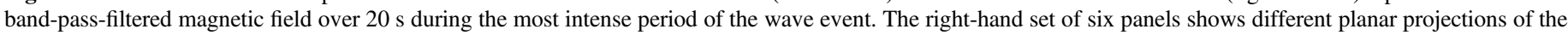

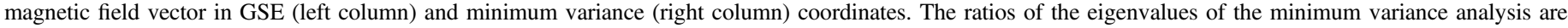

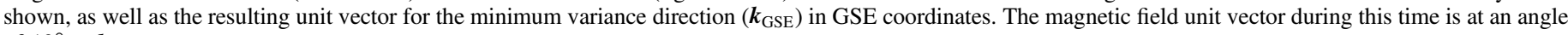
of $13^{\circ}$ to $\boldsymbol{k}_{\mathrm{GSE}}$.

the distribution occurs where the positive gradients become negative (red changing to blue with increasing $V$ ). A secondary positive gradient can be seen as a pink and white stripe between 309.45 and 309.85 at speeds between 350 and $400 \mathrm{~km} \mathrm{~s}^{-1}$. The positive gradient in the proton distribution between the core and the secondary peak region is a source of free energy and coincides with the most intense wave activity identified in the magnetic field data.

\subsubsection{Linear Dispersion Analysis}

Next, we test the stability of the proton distribution function using linear Vlasov theory to find which, if any, modes are able to grow using a range of angles between the wavevector and magnetic field direction, $\theta_{k B}$, under the conditions found in the solar wind. The growing modes are the modes that are most likely to be present.

The linearized kinetic dispersion equation for electromagnetic waves in a uniform, finite-temperature plasma immersed in a constant mean magnetic field is given by

$$
\boldsymbol{D}\left(\boldsymbol{k}, \omega, \theta_{k B} ; p p\right) \cdot \delta \boldsymbol{E}(\boldsymbol{k}, \omega)=0,
$$

where $\boldsymbol{D}\left(\boldsymbol{k}, \omega, \theta_{k B} ; p p\right)$ is the dispersion tensor for a given wavevector $\boldsymbol{k}$, frequency $\omega$, magnetic field direction $\theta_{k B}$, and other plasma parameters $p p$, including drifts, anisotropies, temperatures, etc. $\delta \boldsymbol{E}(\boldsymbol{k}, \omega)$ are the electric field eigenmodes (fluctuations) in the plasma frame. The Appendix of Viñas et al. (2000) explains the theoretical approach in detail. Here we only note that the procedure to calculate the linear eigenmodes is well established, and we use the code already developed and extensively tested (Viñas et al. 2000) to solve for the unstable linear eigenmodes. Figure 6 presents growth rate calculations (Viñas et al. 1984, 2000) of Alfvén ion-cyclotron and fast branch waves for Wind/SWE solar wind observations at three different times on DOY 309. The plasma parameters used for each analysis are taken from the Wind observations and are shown in Table 1.

The results of the stability analysis of the proton distribution function are shown in Figure 6. In the first time interval (left column) at the onset of the wave activity around 309.4540 there is one unstable mode driven by the temperature anisotropy of the core proton population that may generate Alfvén-proton-cyclotron waves. The second time interval is during the most intense wave activity of the event at 309.5095 (middle column of Figure 6) and has two unstable modes driven by the temperature anisotropy of the core (top panel) and the beam (bottom panel) distributions, both of which are proton-cyclotron modes. In the final time period studied, 309.6500 , the core distribution is unstable to the magnetosonic mode (firehose instability), but this is an order of magnitude slower than the cyclotron instability of the other time periods.

These linear stability analyses show that the temperature anisotropies in the core and beam during the event are likely responsible for generating the cyclotron waves. The value of the temperature anisotropy of both the core and beam is not particularly high for the solar wind, which is observed to have a very variable level of anisotropy (Kasper et al. 2002; Hellinger et al. 2006; Matteini et al. 2007; Bale et al. 2009; Hellinger \& Trávníček 2014). The emergence of ion-cyclotron instabilities in the dispersion relation is due to the combined effects of thermal anisotropy and drift velocity (Gary et al. 1984; Gomberoff \& Valdivia 2003; Moya et al. 2014). In particular, the instability is driven by the anisotropy, and the drift produces an amplification to the wave growth. With no anisotropy there would be no waves, but the same anisotropy with no drift produces a smaller growth rate of the unstable modes. The beam itself is not drifting fast enough $\left(V_{d} / V_{A} \sim 1\right)$ 


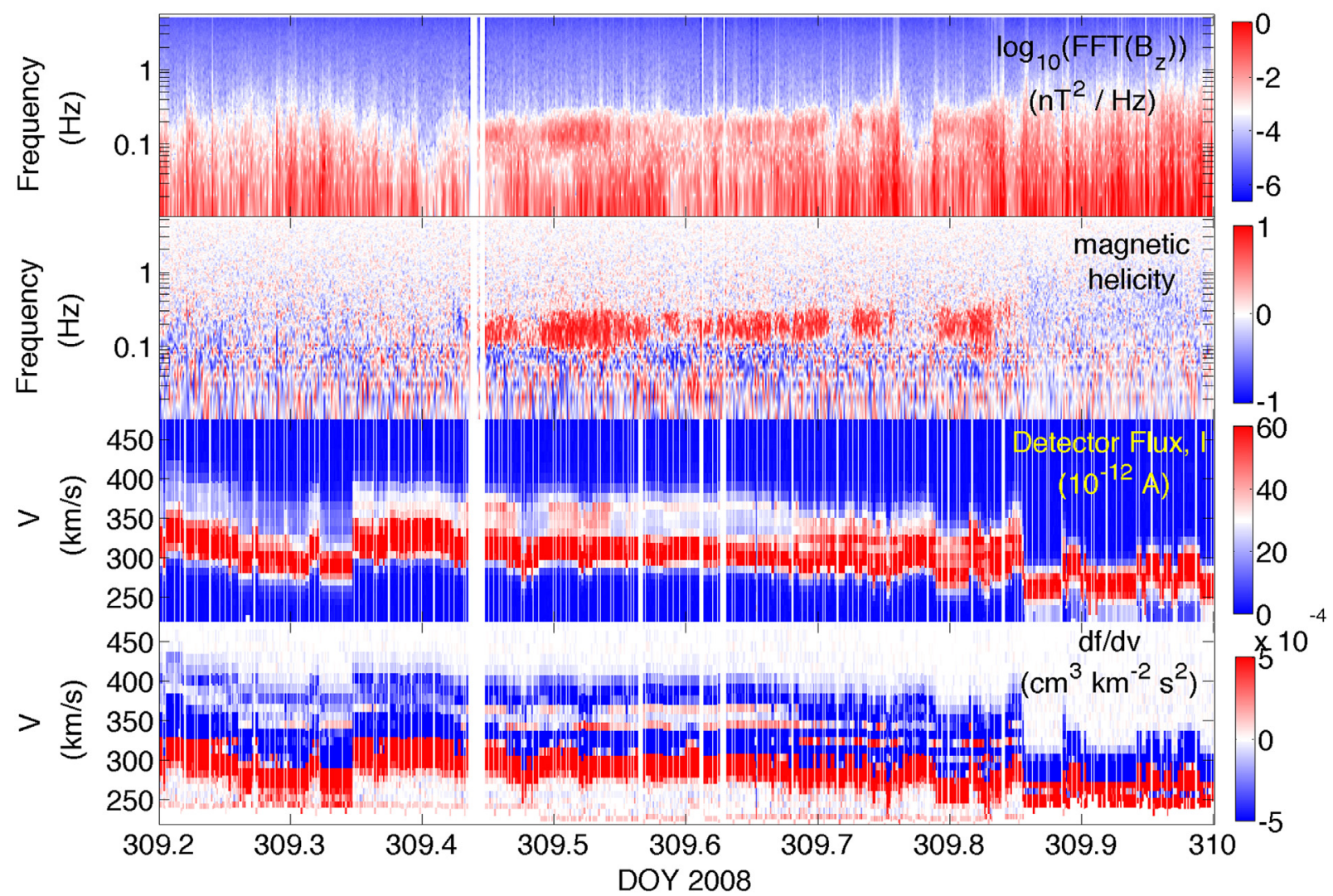

Figure 5. The $92 \mathrm{~s}$ magnetic field spectra for DOY 309.2-310 are plotted in the top panel and the associated magnetic helicity, $\sigma_{m}$, in the second panel, with frequency measured in the spacecraft frame. As in Figure 1, wave activity can be seen between DOY 309.45 and 309.85. The third panel shows the 1D slice of the charge flux in the SWE detector most closely aligned with $B_{0}$ during each $92 \mathrm{~s}$ interval. A particularly dense secondary peak can be seen drifting at higher speeds than the core distribution over the same time interval. The bottom panel shows the inferred gradient of the proton distribution function. Between DOY 309.45 and 309.85 a positive gradient (red) can be seen between speeds of 350 and $400 \mathrm{~km} \mathrm{~s}^{-1}$.

to drive a beam instability (for example, Gary et al. [1984] use $V_{d} / V_{A}$ of up to 30 to produce rapidly growing waves).

Finally, we investigate the link between the waves and proton beam in Figure 7 by plotting the amplitude of the magnetic field spectrum at the wave spectral peak against the kinetic energy density of the beam in the frame of the core distribution. We identify the peak amplitude by finding the frequency $f_{\text {sc }}^{-}$(Equation (5)) for each $92 \mathrm{~s}$ interval and then finding the local maximum value of the trace of the magnetic field Fourier transform for that $92 \mathrm{~s}$ in the range $f_{\mathrm{sc}}^{-} / 10<f<f_{\mathrm{sc}}^{-}$. The corresponding magnetic energy is then

$$
E_{\mathrm{wave}}=\frac{\sum_{i=j} P_{i j}^{\max } f^{\max }}{2 \mu_{0}} .
$$

Time periods in which the core or beam is unstable to the proton-cyclotron instability (defined as having a linear growth rate higher than $10^{-3} \Omega_{p}$ as defined in Hellinger et al. 2006) are plotted as red squares (core) and blue circles (beam). There is a general trend in the plot with higher beam kinetic energy correlated with higher-amplitude waves; however, unstable distributions are more likely to have higher amplitudes than stable periods with the same beam kinetic energy. This reinforces the interpretation of the cyclotron wave storm as a result of the unstable, anisotropic proton distributions with a strong dependence on the presence of the beam.

\section{SUMMARY AND CONCLUSIONS}

We have shown that the audification of high-resolution, long-time-series magnetic field data from the Wind spacecraft can be used to identify proton-cyclotron wave storms in the solar wind. We present a detailed case study of a particularly clear wave event, identified from listening to audified MFI data. Figures 1 and 2 show a summary of the solar wind conditions during 2008 November 4 (DOY 309). For most of the day the solar wind magnetic field is radial and points back toward the Sun. During this interval, a dense proton beam occurs, drifting close to the Alfvén speed ahead of the core of the distribution. Simultaneous with the appearance of the beam, waves are seen in the magnetic field data and heard in the audio of the $B_{z}$ GSE component; the waves have amplitudes up to $0.3 \mathrm{nT}$ and, although intermittent, last for more than $8 \mathrm{hr}$.

Detailed investigation of these waves shows them to be sinusoidal and transverse with a minimum variance direction that is likely closely aligned with the magnetic field (Figures 3 and 4). The frequency of the waves is found to be just below the Doppler-shifted proton gyrofrequency in the spacecraft frame. These are all characteristics of Alfvén proton-cyclotron waves. Furthermore, investigation of the proton distribution functions measured during the event shows that there is a significant secondary proton population drifting parallel to the magnetic field and there are intermittent patches of temperature anisotropy, with $T_{\perp}>T_{\|}$, for both the core and the beam distributions. The secondary peak present in the proton distribution function parallel to the magnetic field during the 

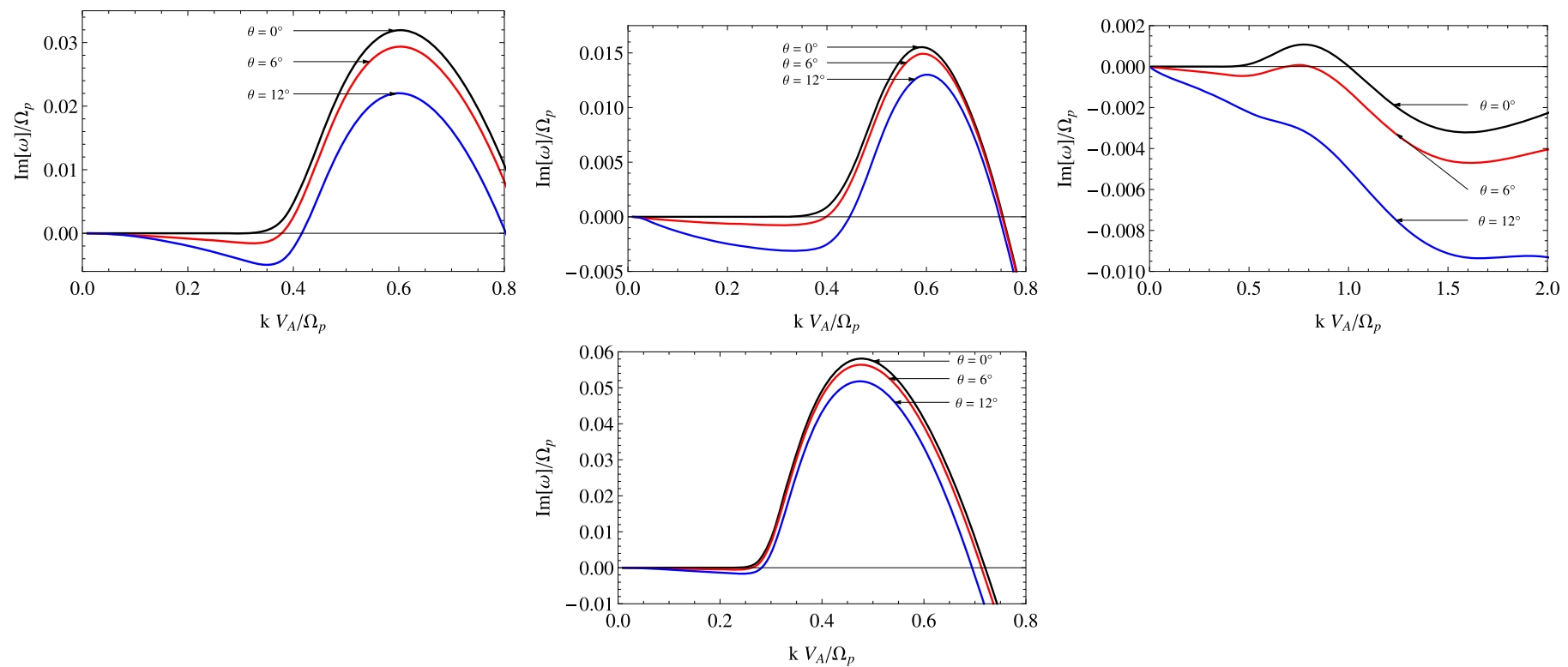

Figure 6. Left panel: 309.454 , growing core-distribution proton-cyclotron mode; middle panels: 309.5095 , growing core (top) and beam distribution protoncyclotron (bottom) modes; right panel: 309.65, growing magnetosonic mode. In all plots each curve represents the solution of Equation (7) at a particular fixed angle between wavevector and magnetic field direction $\theta_{k B}$. The plasma frame frequency of the growing modes is lower than the proton gyrofrequency, qualitatively matching the results in Figures 1 and 3 of the wave-amplitude peak being just below the proton gyrofrequency in the spacecraft frame.

Table 1

Physical Parameters Measured during Three Different $92 \mathrm{~s}$ Time Periods during the Wave and Beam Event

\begin{tabular}{lccccc}
\hline \hline Interval (DOY) & Population & $n / n_{0}$ & $\beta_{\|}$ & $T_{\perp} / T_{\|}$ & $V_{d}\left(\mathrm{~km} \mathrm{~s}^{-1}\right)$ \\
\hline 309.4540 & Core & $0.77 \pm 0.06$ & $0.40 \pm 0.04$ & $2.3 \pm 0.3$ & $47 \pm 3$ \\
& Beam & $0.23 \pm 0.02$ & $0.55 \pm 0.05$ & $0.9 \pm 0.2$ & $V_{A}\left(\mathrm{~km} \mathrm{~s}^{-1}\right)$ \\
\hline 309.5095 & Core & $0.63 \pm 0.03$ & $0.22 \pm 0.02$ & $2.3 \pm 0.2$ & $40 \pm 3$ \\
& Beam & $0.37 \pm 0.02$ & $0.65 \pm 0.04$ & $2.9 \pm 0.2$ & $41 \pm 2$ \\
\hline 309.6500 & Core & $0.71 \pm 0.03$ & $0.27 \pm 0.02$ & $0.75 \pm 0.06$ & $49 \pm 2$ \\
& Beam & $0.29 \pm 0.01$ & $0.61 \pm 0.03$ & $0.61 \pm 0.05$ & $49 \pm 2$ \\
\hline
\end{tabular}

Note. "Core" refers to the denser, slower proton population and "Beam" to the faster population. $n / n_{0}$ is the ratio of the number density of particles fit with each biMaxwellian ( $n_{c}$ for the core and $n_{b}$ for the beam) to the total of both, $n_{0}=n_{c}+n_{b} . \beta_{\|}=2 \mu_{0} n_{c, b} k_{B} T_{\|} /|B|^{2}$ is the field-parallel plasma beta for each population separately, and $T_{\perp} / T_{\mid l}$ is the temperature anisotropy from each of the two bi-Maxwellian fits separately. $V_{d}$ is the drift speed of the beam relative to the core, parallel to the local magnetic field direction, and $V_{A}=|B| / \sqrt{\mu_{o} m_{p} n_{0}}$ is the local Alfvén speed.

majority of the wave event has a slight positive gradient between the core and the beam populations in the distribution function (Figure 5). A linear Vlasov analysis using proton distributions observed during three different periods at the onset, the most intense part, and the middle of the wave event (Table 1) show that the proton distribution functions are mostly unstable to the proton-cyclotron mode owing to the temperature anisotropy $\left(T_{\perp}>T_{\|}\right)$(Figure 6) even though the anisotropy values are not particularly extreme. The amplitude of the waves correlates with the kinetic energy of the beam in the frame of the core distribution, and unstable distributions are more likely to have higher amplitudes (Figure 7). These combined results indicate that the beam is essential to the growth of the waves; the temperature anisotropy of either population alone would not have resulted in as rapidly growing cyclotron waves.

We conclude that the unstable proton distribution, comprising anisotropic core and beam populations, caused the longduration, large-amplitude waves. This conclusion then leaves us with an open question: where did the beam come from? Since the distributions are unstable, they should evolve toward stability rapidly compared to the expansion time of the solar wind, and so it is unlikely that the beam has traveled all the way from the Sun. The beam begins and ends at magnetic discontinuities with highly radial magnetic field in between and is found at the trailing edge of a fast stream. This radial field feature is similar to other events that have been described as signatures of fast-slow wind boundaries and are hypothesized to be formed by footpoint motions of magnetic field lines in the chromosphere or interchange reconnection in the corona (Gosling \& Skoug 2002; Schwadron 2002; Riley \& Gosling 2007; Orlove et al. 2013).

The beam (or indeed the core; since the beam is the same speed as the preceding and following solar wind, one could interpret the denser core population as a slow dense population added to the faster stream) could then be described as a second proton population formed by the interpenetration of solar wind from different sources with the different speed solar wind streams mixing through a (previously) reconnected set of field lines. The beam and waves would then be a remnant of whatever process operates in the corona to produce such unusually long and smooth radial field intervals with the waves and ion distribution functions remaining in a quasi-stationary 


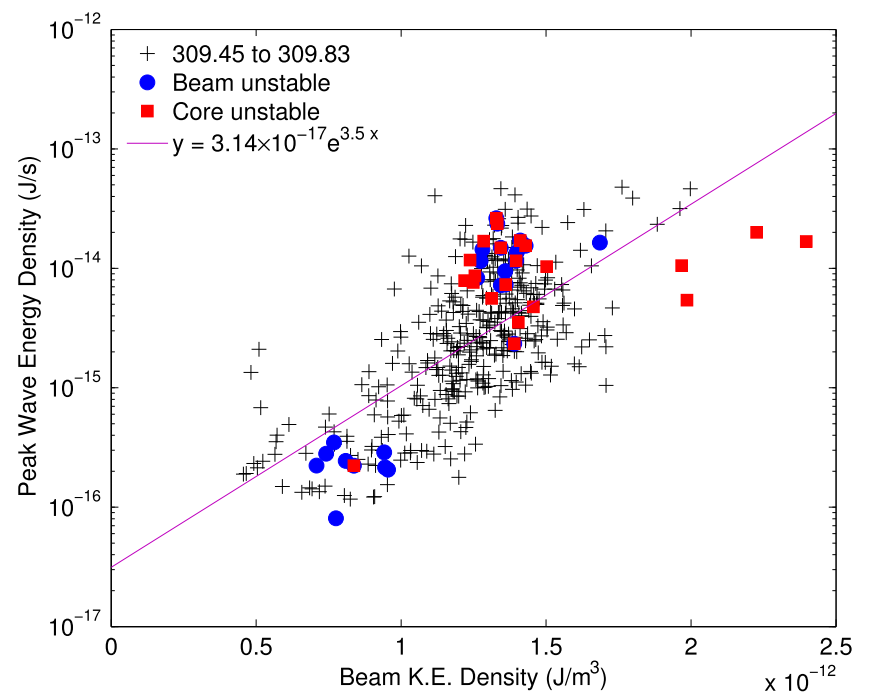

Figure 7. Relationship between beam kinetic energy density and magnetic field wave energy density with temperature-anisotropy-unstable proton distributions for the core (red squares) and beam (blue circles) highlighted. An exponential curve fit to the data with a linear least-squares method in log-linear space is shown (purple line).

state. As the solar wind expands and the Alfvén speed decreases, waves may be continuously generated to reshape the distribution function, keeping the beam around the Alfvén speed ahead of the core. A similar magnetic structure is observed on the same day in situ by the STEREO A spacecraft, which is $41^{\circ}$ ahead of the Earth in its orbit; thus, the feature is a very broad structure at $1 \mathrm{AU}$.

Overall, the presence of the beam and the temperature anisotropy in both the core and the beam populations are associated with the Alfvén-proton-cyclotron waves. Ioncyclotron wave events have been observed with some regularity in the solar wind (Jian et al. 2009, 2010), particularly when the magnetic field is close to radial, and in the trailing edges of fast wind streams (Jian et al. 2014). It seems likely that, in this case, the local proton distribution function is involved in generating the cyclotron waves, and if this is the case for the cyclotron wave storm events observed elsewhere, the implication is that proton beams, or at least temperature anisotropies, are quite common. This is indeed supported by in situ data surveys of the properties of proton distribution functions (Kasper et al. 2002; Bale et al. 2009). Thus, it seems possible that proton-cyclotron waves in the solar wind are, in general, locally excited by unstable distribution functions.

R.T.W. was supported by the NASA Postdoctoral Program at the Goddard Space Flight Center, administered by Oak Ridge Associated Universities. R.T.W. and D.A.R. acknowledge a Heliophysics Guest Investigator grant to NASA GSFC for support. P.S.M. thanks the Conicyt-Becas Chile Postdoctoral Fellowship for financial support. L.K.J. was supported by NASA grant NNX13AI65G. Wind data were obtained from the SPDF Web site http://spdf.gsfc.nasa.gov. The authors thank L. Matteini for useful discussions.

\section{REFERENCES}

Alexander, R. L., Gilbert, J. A., Landi, E., et al. 2011, in Proc. 17th Int. Conf. Aud. Disp., ICAD

Alexander, R. L., O’Modhrain, S., Roberts, D. A., et al. 2014, JGRA, 119,5259

Bale, S. D., Kasper, J. C., Howes, G. G., et al. 2009, PhRvL, 103, 211101

Barkhausen, H. 1919, ZPhy, 29, 401

Barkhausen, H. 1930, in Proc. Inst. of Radio Engineers, 18, 1155

Gary, S. P. 1993, Theory of Space Plasma Microinstabilities (Cambridge: Cambridge Univ. Press)

Gary, S. P., Smith, C. W., Lee, M. A., et al. 1984, PhFl, 27, 1852

Goldstein, M. L., Roberts, D. A., \& Matthaeus, W. H. 1995, ARA\&A, 33, 283

Gomberoff, L., \& Valdivia, J. A. 2003, JGR, 108, A1

Gosling, J. T., \& Skoug, R. M. 2002, JGR, 107, 1327

Hellinger, P., \& Trávníček, P. M. 2014, ApJL, 784, L15

Hellinger, P., Trávníček, P. M., Kasper, J. C., \& Lazarus, A. J. 2006, GeoRL, 33, L09101

Horbury, T. S., Forman, M. A., \& Oughton, S. 2008, PhRvL, 101, 175005

Isenberg, P. A. 2012, PhPl, 19, 032116

Jian, L. K., Russell, C. T., Luhmann, J. G., et al. 2009, ApJL, 701, L105

Jian, L. K., Russell, C. T., Luhmann, J. G., et al. 2010, JGR, 115, A12115

Jian, L. K., Wei, H. Y., Russell, C. T., et al. 2014, ApJ, 786, 123

Kasper, J. C., Lazarus, A. J., \& Gary, S. P. 2002, GeoRL, 29, 1839

Kiyani, K. H., Chapman, S. C., Sahraoui, F., et al. 2013, ApJ, 763, 10

Kramer, G. 1994, in An Introduction to Auditory Display, ed. G. Kramer (Reading, MA: Addison-Wesley), 1

Kramer, G., Walker, B., Bonebright, T., et al. 2010, Sonification Report: Status of the Field and Research Agenda (Department of Psychology: Faculty Publications) http://digitalcommons.unl.edu/psychfacpub/444

Landi, E., Alexander, R. L., Gruesbeck, J. R., et al. 2012, ApJ, 744, 100 Lepping, R. P., Acuña, M. H., Burlaga, L. F., et al. 1995, SSRv, 71, 207 Matteini, L., Landi, S., Hellinger, P., et al. 2007, GeoRL, 34, L20105

Moya, P. S., Navarro, R., Viñas, A. F., Muñoz, V., \& Valdivia, J. A. 2014, ApJ, 781, 76

Ogilvie, K. W., Chornay, D. J., Fritzenreiter, R. J., et al. 1995, SSRv, 71, 55

Orlove, S. T., Smith, C. W., Vasquez, B. J., et al. 2013, ApJ, 774, 15

Podesta, J. J. 2009, ApJ, 698, 986

Podesta, J. J., \& TenBarge, J. M. 2012, JGR, 117, A10106

Richardson, J. D., \& Smith, C. W. 2003, GeoRL, 30, 5

Riley, P., \& Gosling, J. T. 2007, JGR, 112, A06115

Scarf, F. L., Gurnett, D. A., Kurth, W. S., \& Poynter, R. L. 1982, Sci, 215, 587 Schwadron, N. A. 2002, GeoRL, 29, 14

Servidio, S., Osman, K. T., Valentini, F., et al. 2014, ApJL, 781, L27

Smith, E. J., Holzer, R. E., \& Russell, C. T. 1969, JGR, 74, 3027

Viñas, A. F., Goldstein, M. L., \& Acuña, M. H. 1984, JGR, 89, 3762

Viñas, A. F., Wong, H. K., \& Klimas, A. J. 2000, ApJ, 528, 509

Wicks, R. T., Forman, M. A., Horbury, T. S., \& Oughton, S. 2012, ApJ, 746, 103

Wicks, R. T., Horbury, T. S., Chen, C. H. K., \& Schekochihin, A. A. 2010, MNRAS, 407, L31

Wicks, R. T., Matteini, L., Horbury, T. S., Hellinger, P., \& Roberts, D. A. 2013, in AIP Conf. Proc. 1539, Proc. 13th Int. Solar Wind Conf., ed. G. P. Zank et al. (Melville, NY: AIP), 303

Wilson, L. B., Cattell, C. A., Kellogg, P. J., et al. 2009, JGRA, 114

Wilson, L. B., Koval, A., Sibeck, D. G., et al. 2013, JGRA, 118, 957 